Gudelius, D., et al., 2021, Crustal fluids cause strong Lu-Hf fractionation and Hf-Nd-Li isotopic provinciality in the mantle of continental subduction zones: Geology, https://doi.org/10.1130/G49317.1

\title{
Crustal fluids cause strong Lu-Hf fractionation and Hf-Nd-Li isotopic provinciality in the mantle of continental subduction zones
}

\author{
Dominik Gudelius, Sonja Aulbach, Hans-Michael Seitz, and Roberto Braga.
}

\section{SUPPLEMENTARY MATERIAL 1: ANALYTICAL METHODS}

Specimens of eleven peridotites and pyroxenites from the Ulten Zone were cut into representative blocks and were processed as whole rock powders with an agate mill after the abrasion of surfaces to remove metal contamination from the saw. All laboratory work was performed inside a class 100 clean room equipped with metal-free, laminar-flow fume hoods. Additionally, all acids used in this study were double distilled and diluted using ultra-pure water $($ MilliQ) with a resistivity $\geq 18 \mathrm{M} \Omega$. All analyses were performed in the Geoscience Institute at Goethe-University, Frankfurt.

\section{Whole rock Lu-Hf and Sm-Nd isotope analysis}

The samples from the Ulten Zone, replicates of BIR-1 and UB-N standards and full procedural blanks were digested in screwtop Teflon-beakers by using a mixture of $1 \mathrm{ml} 14 \mathrm{M}$ $\mathrm{HNO}_{3}, 3 \mathrm{ml} 28 \mathrm{M} \mathrm{HF}$ and 100-500 $\mu 1$ 10M HClO 4 (100 $\mu 1 \mathrm{HClO}_{4}$ per $50 \mathrm{mg}$ sample). Before digestion, the samples were spiked with ${ }^{176} \mathrm{Lu}^{180}{ }^{18 f}$ and ${ }^{149} \mathrm{Sm}-{ }^{150} \mathrm{Nd}$ tracers. The separation of $\mathrm{Lu}, \mathrm{Hf}, \mathrm{Sm}$ and $\mathrm{Nd}$ was performed using a three-step column chromatography using analytical protocols of Münker et al. (2001), Pin \& Zalduegui (1997) with modification according to Lazarov et al. (2009).

The purified samples of Lu, Hf, Sm and Nd were analyzed separately using multi collector-inductively coupled plasma-mass spectrometry (MC-ICP-MS). For analysis, a Neptune (Thermo-Finnigan) mass spectrometer was used. All analyses were performed by using an Aridus II (Cetac) spray chamber. Repeated measurement of the acid blank (2\% $\mathrm{HNO}_{3}$ ) resulted in detection limits (bg $\left.+3 \times \sigma_{\mathrm{bg}}\right)$ of: 2-216 ppt Hf, 2-30 ppt Lu, 12-35 ppt Nd and $6 \mathrm{ppt} \mathrm{Sm.} \mathrm{Full} \mathrm{procedural} \mathrm{blanks} \mathrm{were:} 0.02 \mathrm{ppb} \mathrm{Hf}, 0.1 \mathrm{ppb} \mathrm{Lu}, 0.01 \mathrm{ppb} \mathrm{Nd}$ and 0.06 ppb Sm. Because these background levels are insignificant compared to the sample concentrations, no correction was applied. Replicate analysis of standard materials yielded the following isotope ratios: ${ }^{176} \mathrm{Hf} /{ }^{177} \mathrm{Hf}$ of $0.283241 \pm 0.000018(2 \sigma ; \mathrm{n}=4)$ for BIR-1 (Bizzarro et al. (2003): $\left.{ }^{176} \mathrm{Hf} /{ }^{177} \mathrm{Hf}=0.283252-0.283281\right) ;{ }^{176} \mathrm{Hf} /{ }^{177} \mathrm{Hf}$ of $0.283245 \pm 0.000032(2 \sigma ; \mathrm{n}$ 
$=$ ) for UB-N; ${ }^{143} \mathrm{Nd} /{ }^{144} \mathrm{Nd}$ of $0.513076 \pm 0.000022(2 \sigma ; \mathrm{n}=4)$ for BIR-1 (Pin et al. (2014):

$\left.{ }^{143} \mathrm{Nd} /{ }^{144} \mathrm{Nd}=0.513074-0.513080\right)$ and ${ }^{143} \mathrm{Nd} /{ }^{144} \mathrm{Nd}$ of $0.512925 \pm 0.000020(2 \sigma ; \mathrm{n}=2)$ for UB-N (Pin \& Zalduegui (1997): $\left.{ }^{143} \mathrm{Nd} /{ }^{144} \mathrm{Nd}=0.512925-0.512926\right)$.

\section{Whole rock $\mathrm{Li}$ isotope analysis}

About $50 \mathrm{mg}$ of the whole rock powders were digested at $170^{\circ} \mathrm{C}$ on hotplate in screwtop Teflon beakers using a mixture of $1 \mathrm{ml}$ of $6 \mathrm{M} \mathrm{HNO}_{3}$ and $1 \mathrm{ml} 28 \mathrm{M} \mathrm{HF}$. Purification of Li was performed using the analytical protocol described by Seitz et al. (2004). Lithium isotope ratios were obtained by MC-ICP-MS using the Neptune (Thermo-Finnigan) equipped with an Aridus (Cetac) spray chamber. The measurement was carried out by standard-sampling bracketing method using a 10 ppb L-SVEC (NIST) standard solution, which also was used to determine Li concentrations. For each sample solution, 3-4 analyses were performed and averaged. Analysis of standard materials yielded the following values: JB-2: $\delta^{7} \mathrm{Li}=4.2 \% \pm$ $0.8 \%$ o $(1 \sigma ; \mathrm{n}=6) ; 8$ ppm Li; UB-N: $\delta^{7} \mathrm{Li}=-2.7 \%$ \% $\pm .4 \%$ o $(1 \sigma ; \mathrm{n}=6) ; 24.5$ ppm Li $\left(\delta^{7} \mathrm{Li}=\right.$ $\left[\left({ }^{7} \mathrm{Li} /{ }^{6} \mathrm{Li}\right)_{\text {sample }} /\left({ }^{7} \mathrm{Li} /{ }^{6} \mathrm{Li}\right)_{\mathrm{L}-\mathrm{SVEC}}\right.$ standard -1$\left.] \times 1000\right)$. These results are in accordance with previously reported values compiled in the GeoReM database (Jochum et al., 2005): JB-2: $\delta^{7} \mathrm{Li}=3.6$ to $5.7 \%$; 7.2 to $8.9 \mathrm{ppm} \mathrm{Li}$; UB-N: $\delta^{7} \mathrm{Li}=-3$ to $-1.8 \%$; 20.6 to $32 \mathrm{ppm} \mathrm{Li}$. 


\section{SUPPLEMENTARY MATERIAL 2: ISOTOPIC DATA}

Bulk rock Lu-Hf, Sm-Nd, and Li isotope systematics for peridotites and pyroxenites of the Ulten Zone

\begin{tabular}{|c|c|c|c|c|c|c|c|c|c|c|c|c|c|c|c|c|c|}
\hline Sample & $\begin{array}{l}\text { wt } \\
(\mathrm{g})\end{array}$ & ${ }^{176} \mathrm{Lu} /{ }^{177} \mathrm{Hf}$ & ${ }^{176} \mathrm{Hf} /{ }^{177} \mathrm{Hf}$ & $2 \sigma$ & $\varepsilon \mathrm{Hf}^{\mathrm{a}, \mathrm{b}}$ & $\varepsilon \mathrm{Hf}_{330^{c}} \mathrm{c}$ & $\begin{array}{l}\text { Lu } \\
(\mathrm{ppm})\end{array}$ & $\begin{array}{l}\text { Hf } \\
(\mathrm{ppm})\end{array}$ & ${ }^{147} \mathrm{Sm} /{ }^{144} \mathrm{Nd}$ & ${ }^{143} \mathrm{Nd} /{ }^{144} \mathrm{Nd}$ & $2 \sigma$ & $\varepsilon \mathrm{Nd}^{\mathrm{a}, \mathrm{b}}$ & $\varepsilon \mathrm{Nd}_{330^{\mathrm{C}}}$ & $\begin{array}{l}\text { Sm } \\
\text { (ppm) }\end{array}$ & $\begin{array}{l}\mathbf{N d} \\
(\mathrm{ppm})\end{array}$ & $\delta^{7} \mathrm{Li}^{\mathrm{d}}$ & Li \\
\hline \multicolumn{18}{|c|}{ coarse-grained spinel-peridotites with coronitic garnet } \\
\hline UN1 & 0.21 & 0.11651 & 0.28379 & 0.000080 & 35.9 & 17.6 & 0.047 & 0.058 & 0.10622 & 0.51269 & 0.000009 & 1.01 & 4.76 & 0.243 & 1.389 & 7.2 & 5.5 \\
\hline UN2 & 0.20 & 0.16225 & 0.28393 & 0.000130 & 41.1 & 12.8 & 0.066 & 0.059 & 0.36272 & 0.51345 & 0.000022 & 15.9 & 8.9 & 0.139 & 0.232 & 1.7 & 1.4 \\
\hline \multicolumn{18}{|c|}{ fine-grained garnet-amphibole peridotites } \\
\hline UN3 & 0.21 & 0.05903 & 0.28462 & 0.000156 & 65.3 & 59.7 & 0.024 & 0.053 & 0.08149 & 0.51253 & 0.000014 & -2.11 & 2.75 & 0.187 & 1.386 & 1.7 & 1.7 \\
\hline UN6 & 0.20 & 0.08838 & 0.28351 & 0.000122 & 26.2 & 14.1 & 0.034 & 0.055 & 0.09497 & 0.51254 & 0.000010 & -1.82 & 2.46 & 0.249 & 1.583 & 1.0 & 1.7 \\
\hline UN7 & 0.21 & 0.07648 & 0.28355 & 0.000043 & 27.4 & 17.9 & 0.031 & 0.053 & 0.09270 & 0.51253 & 0.000007 & -2.21 & 2.17 & 0.231 & 1.504 & 2.7 & 2.8 \\
\hline UN9 & 0.10 & 0.07130 & 0.28306 & 0.000055 & 10.3 & 1.9 & 0.035 & 0.076 & 0.11703 & 0.51251 & 0.000012 & -2.45 & 0.91 & 0.194 & 1.003 & -1.5 & 3.0 \\
\hline UN14 & 0.05 & 0.01850 & 0.28278 & 0.000025 & 0.2 & 3.5 & 0.040 & 0.305 & 0.11942 & 0.51256 & 0.000007 & -1.61 & 1.64 & 0.477 & 2.416 & -3.7 & 3.6 \\
\hline \multicolumn{18}{|c|}{ fine-grained spinel-amphibole peridotites } \\
\hline UN8 & 0.10 & 0.01238 & 0.28271 & 0.000036 & -2.3 & 2.3 & 0.016 & 0.173 & 0.12234 & 0.51250 & 0.000010 & -2.66 & 0.47 & 0.346 & 1.708 & -2.8 & 3.8 \\
\hline UN16 & 0.24 & na & na & na & na & na & na & na & 0.08834 & 0.51232 & 0.000009 & -6.26 & -1.70 & 0.109 & 0.745 & 2.1 & 4.6 \\
\hline \multicolumn{18}{|c|}{ fine-grained garnet-amphibole pyroxenites } \\
\hline UN10 & 0.06 & 0.03986 & 0.28295 & 0.000023 & 6.2 & 4.8 & 0.110 & 0.380 & 0.10939 & 0.51251 & 0.000011 & -2.47 & 1.21 & 1.101 & 6.085 & -4.2 & 3.2 \\
\hline UN11 & 0.06 & 0.04715 & 0.28308 & 0.000019 & 10.8 & 7.8 & 0.120 & 0.338 & 0.11072 & 0.51250 & 0.000007 & -2.67 & 0.95 & 0.970 & 5.298 & 0.3 & 2.9 \\
\hline \multicolumn{18}{|c|}{ Standards } \\
\hline BIR-1 & 0.05 & 0.06099 & 0.28324 & 0.000018 & 16.6 & & 0.250 & 0.582 & 0.26961 & 0.51308 & 0.000022 & 8.55 & & 1.051 & 2.356 & & \\
\hline UB-N & 0.10 & 0.05432 & 0.28325 & 0.000032 & 16.7 & & 0.047 & 0.120 & 0.21211 & 0.51293 & 0.000020 & 5.60 & & 0.205 & 0.584 & -2.7 & 24.5 \\
\hline JB-2 & & & & & & & & & & & & & & & & 4.2 & 8.0 \\
\hline
\end{tabular}

$2 \sigma=$ twofold absolute standard error, na $=$ not available, $w \mathrm{t}=$ weight of digested sample powder

per 10,000 deviation from chondritic mantle (present-day)

Chondritic mantle (CHUR) of Bouvier et al. (2008)

${ }^{c} \varepsilon \mathrm{Hf}$ and $\varepsilon N d$ values at $330 \mathrm{Ma}$, the age of the HP stage of the Ulten Zone (Tumiati et al., 2003). $\lambda^{176} \mathrm{Lu}=1.876 * 10^{-11}$ (Söderlund et al., 2004); $\lambda^{147} \mathrm{Sm}=6.524 * 10^{-12}$ (Villa et al., 2020)

${ }^{\mathrm{d}} \delta^{7} \mathrm{Li}$ indicates per 1,000 deviation of ${ }^{7} \mathrm{Li} /{ }^{\circ} \mathrm{Li}$ from L-SVEC standard 


\section{References}

Bizzarro, M., Baker, J.A., and Ulfbeck, D., 2003, A New Digestion and Chemical Separation Technique for Rapid and Highly Reproducible Determination of Lu/Hf and Hf Isotope Ratios in Geological Materials by MC-ICP-MS: Geostandards and Geoanalytical Research, v. 27, no. 2, p. 133-145, doi: 10.1111/j.1751908X.2003.tb00641.x.

Bouvier, A., Vervoort, J.D., and Patchett, P.J., 2008, The Lu-Hf and Sm-Nd isotopic composition of CHUR: Constraints from unequilibrated chondrites and implications for the bulk composition of terrestrial planets: Earth and Planetary Science Letters, v. 273, 1-2, p. 48-57, doi: 10.1016/j.eps1.2008.06.010.

Jochum, K.P., Nohl, U., Herwig, K., Lammel, E., Stoll, B., and Hofmann, A.W., 2005, GeoReM: A New Geochemical Database for Reference Materials and Isotopic Standards: Geostandards and Geoanalytical Research, v. 29, no. 3, p. 333-338, doi: 10.1111/j.1751-908X.2005.tb00904.X.

Lazarov, M., Brey, G.P., and Weyer, S., 2009, Time steps of depletion and enrichment in the Kaapvaal craton as recorded by subcalcic garnets from Finsch (SA): Earth and Planetary Science Letters, v. 279, 1-2, p. 1-10, doi: 10.1016/j.epsl.2008.12.015.

Münker, C., Weyer, S., Scherer, E., and Mezger, K., 2001, Separation of high field strength elements ( $\mathrm{Nb}, \mathrm{Ta}, \mathrm{Zr}, \mathrm{Hf})$ and Lu from rock samples for MC-ICPMS measurements: Geochemistry, Geophysics, Geosystems, v. 2, no. 12, doi: 10.1029/2001GC000183. Pin, C., Gannoun, A., and Dupont, A., 2014, Rapid, simultaneous separation of Sr, Pb, and $\mathrm{Nd}$ by extraction chromatography prior to isotope ratios determination by TIMS and MC-ICP-MS: J. Anal. At. Spectrom., v. 29, no. 10, p. 1858-1870, doi: 10.1039/c4ja00169a. 
Pin, C., and Zalduegui, J.S., 1997, Sequential separation of light rare-earth elements, thorium and uranium by miniaturized extraction chromatography: Application to isotopic analyses of silicate rocks: Analytica Chimica Acta, v. 339, 1-2, p. 79-89, doi: 10.1016/S0003-2670(96)00499-0.

Seitz, H.-M., Brey, G.P., Lahaye, Y., Durali, S., and Weyer, S., 2004, Lithium isotopic signatures of peridotite xenoliths and isotopic fractionation at high temperature between olivine and pyroxenes: Chemical Geology, v. 212, 1-2, p. 163-177, doi: 10.1016/j.chemgeo.2004.08.009.

Söderlund, U., Patchett, P., Vervoort, J.D., and Isachsen, C.E., 2004, The ${ }^{176} \mathrm{Lu}$ decay constant determined by $\mathrm{Lu}-\mathrm{Hf}$ and $\mathrm{U}-\mathrm{Pb}$ isotope systematics of Precambrian mafic intrusions: Earth and Planetary Science Letters, v. 219, 3-4, p. 311-324, doi: 10.1016/S0012-821X(04)00012-3.

Tumiati, S., Thöni, M., Nimis, P., Martin, S., and Mair, V., 2003, Mantle-crust interactions during Variscan subduction in the Eastern Alps (Nonsberg-Ulten zone): Geochronology and new petrological constraints: Earth and Planetary Science Letters, v. 210, 3-4, p. 509-526, doi: 10.1016/S0012-821X(03)00161-4.

Villa, I.M., Holden, N.E., Possolo, A., Ickert, R.B., Hibbert, D.B., and Renne, P.R., 2020, IUPAC-IUGS recommendation on the half-lives of ${ }^{147} \mathrm{Sm}$ and ${ }^{146} \mathrm{Sm}$ : Geochimica et Cosmochimica Acta, v. 285, p. 70-77, doi: 10.1016/j.gca.2020.06.022. 